\author{
D.F. ARENCIBIA-ARREBOLA ${ }^{1}$, \\ L.A. ROSARIO-FERNANDEZ ${ }^{2}$, Y.E. SUAREZ-FERNANDEZ ${ }^{3}$, \\ A. VIDAL-NOVOA ${ }^{4}$ \\ ${ }^{1}$ Finlay Institute, 17 Avenue, Playa Municipality, Havana, Cuba \\ E-mail: darencibia@finlay.edu.cu \\ 2 Institute of Pharmacy and Food Science, Havana, Cuba \\ ${ }^{3}$ Medicine Veterinary Faculty UNAH, Cuba \\ ${ }^{4}$ Faculty of Biology, Havana University, Cuba
}

\section{COMPARISON OF MICRONUCLEI FREQUENCY IN BONE MARROW CELLS OF THREE RAT LINES}

Introduction. Usually for testing genotoxicity of different substances researchers use different lines of rats. Due to genetic differences between lines their results cannot be compared. That is why the correct choosing of model organisms remains the very important problem [1]. Comparative studies of spontaneous and induced genome damages in different lines of rats could provide researchers with efficient way of choosing the most appropriate genetic lines of rats for their tests.

One of popular test of genotoxicity is the micronucleus assay in bone marrow cells. This test is easy to perform and it provides researchers with clear information on the rate of genome damages in bone marrow cells. This approach allows to register in vivo an ability of chemical substances to induce chromosome breaks [2]. The increasing of frequency of polychromatic erythrocytes with micronuclei indicates the respective invreasing of chromosome damages $[2,3]$. The detection of micronucleated erythrocytes can also be performed in species, whose spleen cannot eliminate the micronucleated erythrocytes or species sufficiently sensitive to detect agents that cause numeric or structural chromosome aberrations $[2,3]$. The micronucleus assay must include the positive control with mutagenic substance usually cyclophosphamide [2]. This mutagen is an alquilant agent that forms monoadducts and cross-connections between chains [3] and does not possess the specificity dependent on the cell cycle [4]. The aim of this article is to compare the spontaneous and cyclophosphamide induced micronucleus indexes of Sprague Dawley, Lewis and Wistar rat lines.

Materials and methods. Animals and environmental conditions. Sprague Dawley, Lewis and Wistar rat lines, young adults (6-8 weeks of age) of both sexes with body mass about $180-210 \mathrm{~g}$ at the end of the quarantine were used in our work. They were held under temperature $23 \pm 2{ }^{\circ} \mathrm{C}$, relative humidity $60 \pm 10 \%$ and $12 \mathrm{~h}$ cycles of light-darkness. The access to the water and the food (CENPALAB) was ad libitum. These conditions were common for all groups. We adhered to the ethical principles for the investigation with laboratory animals according to the Canadian Advice of Care Animal [5].

Doses and treatment procedures. All the substances were administered in the «two days» shedule and the concentrations were adjusted weekly according to the body mass increasing. The ani-
C D.F.ARENCIBIA-ARREBOLA, L.A. ROSARIO-FERNANDEZ, Y.E. SUAREZ-FERNANDEZ, A.VIDAL-NOVOA, 2013 
mals were randomly distributed among 5 groups with 10 rats of each sex of each line per group. The first group was used as the negative control (intact animals), the second one was exposed to oral administration of drugs (technique control group). The third experimental group was treated with $2 \%$ Tween 65 (carrying substance 1) which is used as carrier of many nonpolar hydrophobic substances, useful as tensoactive agent [6]; the fourth group was treated with $0.9 \% \mathrm{NaCl}$ (carrying sustance 2), useful as solvent of most hydrophilic substances [7]. Both substances were prepared for 2 hours before using and were applied per os to $2 \mathrm{ml} / \mathrm{kg}$ during a period of 14 days. The fifth group was treated intraperitoneally with cyclophosphamide (Ledoxina ${ }^{\circledR}$. Lemery. CORP) previously dissolved in $0,9 \% \mathrm{NaCl}$, in the dose of $50 \mathrm{mg} / \mathrm{kg}$ [8]. The solution of cyclophosphamide was used immediately after being prepared and applied to animals (in dose $10 \mathrm{ml} / \mathrm{kg}$ ), on the $48^{\text {th }}$ and $24^{\text {th }}$ hours before euthanasia [8].

Clinical observations. We performed two daily clinical observations, during 8:30-10:30 a.m. and during 3:00-4:30 p.m. During each observation we had investigated the general clinical state of the animal, including the palpation for the detection of lesions, possible breathing affectations, of the nervous, cardiovascular, gastrointestinal system, state of the skin, hair, mucous and eyes coloration.

Euthanasia method. All animals were sacrificed with ether. We provided this process in a way that the animals did not show pain signs or suffering.

Bone marrow cells micronucleus assay. The micronucleus assay was performed according to the modified protocol [9, 10]. One femur per animal was extracted and the medullar cavity was washed out with $3 \mathrm{~mL}$ of fetal bovine serum. The obtained cells were centrifuged at $200 \mathrm{~g}$ for $10 \mathrm{~min}$; and the pellet was spreaded onto the slides [9]. The slides (minimum 2 slides per animal) were dried $24 \mathrm{~h}$ under ambient temperature, fixed in absolute methanol (5 min) and stained with $5 \%$ Giemsa for 12-15 min [10]. The analysis was performed by three independent observers, using a microscope Olympus BH-2 (100× lens with immersion).

The polychromatic erythrocytes (PE) and normochromatic erythrocytes (NE) were counted among 2000 cells/animal. The young eryth- rocytes are the PE, microscopically they are observed as blue cells and the normochromatic erythrocytes (adults) are observed as pink [2, 9]. The percent of PE with micronucleus (MN-EP) was calculated in $2000 \mathrm{PE} /$ animal. Later the cytotoxicity index (PE/NE) was calculated for the total population of erythrocytes per group and total number of micronucleus (MN) [10]. In this work we presented the average results of three independent observers.

Statistical analysis. We calculated the average $(\mathrm{X})$ and standard deviation (S.D) of studed variables. The comparisons between controls and cyclophosphamide-treated (CF) groups for the same line, among different lines, sex and observers were performed with ANOVA with significance level $\alpha=0.05$. The categorical variables (total number of micronuclei) were analysed by Chi-Square, with significance level of $\alpha=0.01$. All the analyses were performed with STATISTICA (version 6) (StatSoft, Inc, 2003).

Permission of ethics committee. The authors declare that this work was made on the base of good practices of preclinical laboratory that are present in the national regulation of protocols of research, approved in the Cuban republic. When this research began it was obtained the protocol approval in writing.

Results and discussion. We did not observe any systemic damages of the whole organism of animals. We did not observe also animals with symptoms of intoxication. The damages were noted at the level of somatic cells. It let us conclude that the doses and the schedule of treatments were appropriate.

The groups of cyclophosphamide-treated animals differed from other groups of rats, while groups of negative controle, groups of control of the treatment way and two solvent substances did not differe each from other in the same sex groups and lines [7, 11]. The results obtained for Sprague Dawley rats are in good accordance with our previous works [12, 13].

The low (but significant) intoxication (PE/ NE) was noted for Sprague Dawley rats of both sexes. No differences were found among sexes of the same group and rat line (Table 1) [7, 11]. In other studies differences among sexes were not observed also [14-16].

In Sprague Dawley line the $\mathrm{PE} / \mathrm{NE}$ is equal $1.18 \pm 0.06$; the genotoxicity index was about 
The cytotoxicity indexes (PE/NE) and percentage of PE with micronucleus in bone marrow of Sprague Dawley, Lewis and Wistar rats

\begin{tabular}{|c|c|c|c|c|c|}
\hline Groups & $\mathrm{n}$ & Sex & $\mathrm{MN}^{\mathrm{R}}$ & $\mathrm{PE} / \mathrm{NE}^{\mathrm{T}}$ & $\mathrm{MN}-\mathrm{PE}(\%)^{\mathrm{T}}$ \\
\hline \multicolumn{6}{|c|}{ Sprague Dawley Rats } \\
\hline Negative Control (Intact) & $\begin{array}{l}10 \\
10\end{array}$ & $\begin{array}{l}\mathrm{F} \\
\mathrm{M}\end{array}$ & $\begin{array}{l}28 \mathrm{a} \\
26 \mathrm{a}\end{array}$ & $\begin{array}{l}1,21 \pm 0,05 \\
1,17 \pm 0,04\end{array}$ & $\begin{array}{l}0,20 \pm 0,03 a \\
0,18 \pm 0,04 a\end{array}$ \\
\hline Technique Control & $\begin{array}{l}10 \\
10\end{array}$ & $\begin{array}{l}\mathrm{F} \\
\mathrm{M}\end{array}$ & $\begin{array}{l}32 \mathrm{a} \\
27 \mathrm{ac}\end{array}$ & $\begin{array}{l}1,19 \pm 0,06 a \\
1,20 \pm 0,03 a\end{array}$ & $\begin{array}{l}0,22 \pm 0,01 \mathrm{a} \\
0,19 \pm 0,05 \mathrm{a}\end{array}$ \\
\hline Vehicle Substance 1 & $\begin{array}{l}10 \\
10\end{array}$ & $\begin{array}{l}\mathrm{F} \\
\mathrm{M}\end{array}$ & $\begin{array}{l}29 a \\
30 a\end{array}$ & $\begin{array}{l}1,21 \pm 0,02 \mathrm{a} \\
1,19 \pm 0,04 \mathrm{a}\end{array}$ & $\begin{array}{l}0,20 \pm 0,02 \mathrm{a} \\
0,21 \pm 0,02 \mathrm{a}\end{array}$ \\
\hline Vehicle Substance 2 & $\begin{array}{l}10 \\
10\end{array}$ & $\begin{array}{l}\mathrm{F} \\
\mathrm{M}\end{array}$ & $\begin{array}{l}25 \mathrm{a} \\
22 \mathrm{a}\end{array}$ & $\begin{array}{l}1,18 \pm 0,04 a \\
1,18 \pm 0,06 a\end{array}$ & $\begin{array}{l}0,17 \pm 0,05 \mathrm{a} \\
0,15 \pm 0,06 \mathrm{a}\end{array}$ \\
\hline Cyclophosphamide ${ }^{1}$ & $\begin{array}{l}10 \\
10\end{array}$ & $\begin{array}{l}\mathrm{F} \\
\mathrm{M}\end{array}$ & $\begin{array}{c}250^{* *} \mathrm{a} \\
241^{* *} \mathrm{ac}\end{array}$ & $\begin{array}{l}0,89 \pm 0,04 * a \\
0,90 \pm 0,03^{*} \mathrm{a}\end{array}$ & $\begin{array}{l}1,74 \pm 1,03^{*} \mathrm{a} \\
1,68 \pm 0,92^{*} \mathrm{ac}\end{array}$ \\
\hline
\end{tabular}

Lewis Rats

$\begin{array}{lccccc}\text { Negative Control (Intact) } & 10 & \mathrm{~F} & 46 \mathrm{~b} & 1,10 \pm 0,03 \mathrm{~b} & 0,31 \pm 0,04 \mathrm{~b} \\ \text { Technique Control } & 10 & \mathrm{M} & 48 \mathrm{~b} & 1,11 \pm 0,03 \mathrm{~b} & 0,33 \pm 0,03 \mathrm{~b} \\ & 10 & \mathrm{~F} & 48 \mathrm{~b} & 1,11 \pm 0,02 \mathrm{~b} & 0,33 \pm 0,05 \mathrm{~b} \\ \text { Vehicle Substance } 1 & 10 & \mathrm{M} & 47 \mathrm{~b} & 1,09 \pm 0,03 \mathrm{~b} & 0,32 \pm 0,06 \mathrm{~b} \\ & 10 & \mathrm{~F} & 49 \mathrm{~b} & 1,12 \pm 0,02 \mathrm{~b} & 0,34 \pm 0,06 \mathrm{~b} \\ \text { Vehicle Substance 2 } & 10 & \mathrm{M} & 50 \mathrm{~b} & 1,13 \pm 0,02 \mathrm{~b} & 0,34 \pm 0,05 \mathrm{~b} \\ & 10 & \mathrm{~F} & 42 \mathrm{~b} & 1,10 \pm 0,03 \mathrm{~b} & 0,29 \pm 0,07 \mathrm{~b} \\ \text { Cyclophosphamide }^{1} & 10 & \mathrm{M} & 44 \mathrm{~b} & 1,13 \pm 0,02 \mathrm{~b} & 0,30 \pm 0,07 \mathrm{~b} \\ & 10 & \mathrm{~F} & 295^{* *} \mathrm{~b} & 0,82 \pm 0,02 * \mathrm{~b} & 2,03 \pm 1,48^{*} \mathrm{~b} \\ & 10 & \mathrm{M} & 291^{* * \mathrm{~b}} & 0,81 \pm 0,02 * \mathrm{~b} & 2,00 \pm 1,08^{*} \mathrm{~b}\end{array}$

Wistar Rats

$\begin{array}{lccccc}\text { Negative Control (Intact) } & 10 & \mathrm{~F} & 53 \mathrm{~b} & 1,11 \pm 0,02 \mathrm{~b} & 0,36 \pm 0,03 \mathrm{~b} \\ \text { Technique Control } & 10 & \mathrm{M} & 47 \mathrm{bc} & 1,10 \pm 0,04 \mathrm{~b} & 0,33 \pm 0,02 \mathrm{~b} \\ & 10 & \mathrm{~F} & 54 \mathrm{~b} & 1,12 \pm 0,03 \mathrm{~b} & 0,37 \pm 0,02 \mathrm{~b} \\ \text { Vehicle Substance 1 } & 10 & \mathrm{M} & 49 \mathrm{bc} & 1,14 \pm 0,02 \mathrm{~b} & 0,34 \pm 0,04 \mathrm{~b} \\ & 10 & \mathrm{~F} & 50 \mathrm{~b} & 1,10 \pm 0,03 \mathrm{~b} & 0,34 \pm 0,05 \mathrm{~b} \\ \text { Vehicle Substance 2 } & 10 & \mathrm{M} & 55 \mathrm{bc} & 1,11 \pm 0,03 \mathrm{~b} & 0,38 \pm 0,03 \mathrm{~b} \\ & 10 & \mathrm{~F} & 51 \mathrm{~b} & 1,10 \pm 0,03 \mathrm{~b} & 0,35 \pm 0,03 \mathrm{~b} \\ \text { Cyclophosphamide }^{1} & 10 & \mathrm{M} & 46 \mathrm{bc} & 1,12 \pm 0,04 \mathrm{~b} & 0,32 \pm 0,05 \mathrm{~b} \\ & 10 & \mathrm{~F} & 292^{* * \mathrm{bc}} & 0,79 \pm 0,03^{*} \mathrm{~b} & 2,01 \pm 1,56^{*} \mathrm{~b} \\ & 10 & \mathrm{M} & 298^{* * \mathrm{~b}} & 0,80 \pm 0,02 * \mathrm{~b} & 2,05 \pm 1,16^{*} \mathrm{~b}\end{array}$

Inducation. PE - Polychromatic Erythrocytes. NE - Normochromatic Erythrocytes. MN - Micronucleus. Count in 2000 cells/animal of 10 animal/group/sex/line. ${ }^{\mathrm{T}}$ Analyzed variable using the ANOVA test (X: average; SD: Standard Deviation). ${ }^{\mathrm{R}}$ Analysed variable, $\chi^{2}$ non parametric test. ${ }^{1}$ Intraperiytoneal treatment. ${ }^{*} \mathrm{p}<0,05$ (comparison with the negative control group in the same genetic line and sex, ANOVA test). $* * p<0,01$ (comparison with the negative control group in the same genetic line and sex, Chi Square $\left(\chi^{2}\right)$ non parametric test). $\mathrm{a}, \mathrm{b}-$ Different letters it differs when comparing among lines keeping in mind the same variable in the same experimental group and sex. c - Comparison between sexes in the same experimental group and rat lines. 
Variation of data obtained by three observers

\begin{tabular}{|c|c|c|c|c|c|c|c|c|c|c|}
\hline \multirow{2}{*}{ Groups } & \multirow{2}{*}{ Sex } & \multicolumn{3}{|c|}{$\mathrm{MN}^{\mathrm{R}}$} & \multicolumn{3}{|c|}{$\mathrm{PE} / \mathrm{NE}^{\mathrm{T}}$} & \multicolumn{3}{|c|}{$\mathrm{MN}-\mathrm{PE}(\%)^{\mathrm{T}}$} \\
\hline & & Ob1 & $\mathrm{Ob} 2$ & Ob3 & Ob1 & $\mathrm{Ob} 2$ & Ob3 & Ob1 & $\mathrm{Ob} 2$ & Ob3 \\
\hline \multicolumn{11}{|c|}{ Sprague Dawley Rats } \\
\hline $\mathrm{NC}$ & $\begin{array}{l}\mathrm{F} \\
\mathrm{M}\end{array}$ & $\begin{array}{l}25 a \\
24 a\end{array}$ & $\begin{array}{l}30 a \\
24 a\end{array}$ & $\begin{array}{l}29 a \\
30 a\end{array}$ & $\begin{array}{l}1,26 \mathrm{~b} \\
1,14 \mathrm{a}\end{array}$ & $\begin{array}{l}1,18 \mathrm{a} \\
1,19 \mathrm{a}\end{array}$ & $\begin{array}{l}1,19 \mathrm{a} \\
1,18 \mathrm{a}\end{array}$ & $\begin{array}{l}0,15 \mathrm{a} \\
0,22 \mathrm{a}\end{array}$ & $\begin{array}{l}0,19 \mathrm{a} \\
0,15 \mathrm{~b}\end{array}$ & $\begin{array}{l}0,26 \mathrm{a} \\
0,17 \mathrm{a}\end{array}$ \\
\hline $\mathrm{TC}$ & $\begin{array}{l}\mathrm{F} \\
\mathrm{M}\end{array}$ & $\begin{array}{l}27 a \\
22 a\end{array}$ & $\begin{array}{l}34 a \\
29 a\end{array}$ & $\begin{array}{l}35 \mathrm{~b} \\
30 \mathrm{~b}\end{array}$ & $\begin{array}{l}1,18 \mathrm{a} \\
1,22 \mathrm{a}\end{array}$ & $\begin{array}{l}1,23 \mathrm{a} \\
1,20 \mathrm{a}\end{array}$ & $\begin{array}{l}1,16 \mathrm{a} \\
1,19 \mathrm{a}\end{array}$ & $\begin{array}{l}0,25 \mathrm{a} \\
0,17 \mathrm{a}\end{array}$ & $\begin{array}{l}0,22 \mathrm{a} \\
0,19 \mathrm{a}\end{array}$ & $\begin{array}{l}0,19 \mathrm{a} \\
0,21 \mathrm{a}\end{array}$ \\
\hline VS1 & $\begin{array}{l}\mathrm{F} \\
\mathrm{M}\end{array}$ & $\begin{array}{l}26 a \\
33 a\end{array}$ & $\begin{array}{l}27 \mathrm{a} \\
30 \mathrm{a}\end{array}$ & $\begin{array}{l}34 \mathrm{~b} \\
27 \mathrm{a}\end{array}$ & $\begin{array}{l}1,26 \mathrm{~b} \\
1,16 \mathrm{a}\end{array}$ & $\begin{array}{l}1,19 \mathrm{a} \\
1,20 \mathrm{a}\end{array}$ & $\begin{array}{l}1,18 \mathrm{a} \\
1,21 \mathrm{a}\end{array}$ & $\begin{array}{l}0,16 \mathrm{a} \\
0,22 \mathrm{a}\end{array}$ & $\begin{array}{l}0,20 \mathrm{a} \\
0,24 \mathrm{a}\end{array}$ & $\begin{array}{l}0,24 \mathrm{a} \\
0,17 \mathrm{a}\end{array}$ \\
\hline VS2 & $\begin{array}{l}\mathrm{F} \\
\mathrm{M}\end{array}$ & $\begin{array}{l}20 a \\
20 a\end{array}$ & $\begin{array}{l}29 b \\
19 a\end{array}$ & $\begin{array}{c}26 a b \\
27 b\end{array}$ & $\begin{array}{l}1,17 \mathrm{a} \\
1,15 \mathrm{a}\end{array}$ & $\begin{array}{l}1,18 \mathrm{a} \\
1,20 \mathrm{a}\end{array}$ & $\begin{array}{l}1,19 a \\
1,19 a\end{array}$ & $\begin{array}{l}0,16 \mathrm{a} \\
0,15 \mathrm{a}\end{array}$ & $\begin{array}{l}0,15 \mathrm{a} \\
0,13 \mathrm{a}\end{array}$ & $\begin{array}{l}0,20 \mathrm{a} \\
0,17 \mathrm{a}\end{array}$ \\
\hline $\mathrm{CF}$ & $\begin{array}{l}\mathrm{F} \\
\mathrm{M}\end{array}$ & $\begin{array}{l}241 \mathrm{a} \\
235 \mathrm{a}\end{array}$ & $\begin{array}{c}249 a b \\
248 a\end{array}$ & $\begin{array}{l}260 \mathrm{~b} \\
240 \mathrm{a}\end{array}$ & $\begin{array}{l}0,79 \mathrm{a} \\
0,94 \mathrm{a}\end{array}$ & $\begin{array}{l}0,98 \mathrm{a} \\
0,77 \mathrm{a}\end{array}$ & $\begin{array}{l}0,90 \mathrm{a} \\
0,99 \mathrm{a}\end{array}$ & $\begin{array}{l}1,82 \mathrm{~b} \\
1,55 \mathrm{a}\end{array}$ & $\begin{array}{l}1,56 \mathrm{a} \\
1,62 \mathrm{a}\end{array}$ & $\begin{array}{l}1,84 \mathrm{a} \\
1,87 \mathrm{a}\end{array}$ \\
\hline \multicolumn{11}{|c|}{ Lewis Rats } \\
\hline $\mathrm{NC}$ & $\begin{array}{l}\mathrm{F} \\
\mathrm{M}\end{array}$ & $\begin{array}{l}50 a \\
53 a\end{array}$ & $\begin{array}{l}43 a \\
46 a\end{array}$ & $\begin{array}{l}45 a \\
45 a\end{array}$ & $\begin{array}{l}1,10 \mathrm{a} \\
1,17 \mathrm{~b}\end{array}$ & $\begin{array}{c}1,13 \mathrm{a} \\
1,10 \mathrm{ab}\end{array}$ & $\begin{array}{l}1,07 \mathrm{a} \\
1,06 \mathrm{a}\end{array}$ & $\begin{array}{c}0,29 \mathrm{ab} \\
0,36 \mathrm{a}\end{array}$ & $\begin{array}{l}0,27 \mathrm{a} \\
0,34 \mathrm{a}\end{array}$ & $\begin{array}{l}0,37 b \\
0,29 a\end{array}$ \\
\hline $\mathrm{TC}$ & $\begin{array}{l}\mathrm{F} \\
\mathrm{M}\end{array}$ & $\begin{array}{l}43 a \\
43 a\end{array}$ & $\begin{array}{l}46 a \\
45 a\end{array}$ & $\begin{array}{l}55 \mathrm{a} \\
53 \mathrm{a}\end{array}$ & $\begin{array}{l}1,08 \mathrm{a} \\
1,15 \mathrm{a}\end{array}$ & $\begin{array}{l}1,11 \mathrm{a} \\
1,07 \mathrm{a}\end{array}$ & $\begin{array}{l}1,14 \mathrm{a} \\
1,06 \mathrm{a}\end{array}$ & $\begin{array}{l}0,38 \mathrm{a} \\
0,29 \mathrm{a}\end{array}$ & $\begin{array}{l}0,28 \mathrm{a} \\
0,30 \mathrm{a}\end{array}$ & $\begin{array}{l}0,34 \mathrm{a} \\
0,37 \mathrm{a}\end{array}$ \\
\hline VS1 & $\begin{array}{l}\mathrm{F} \\
\mathrm{M}\end{array}$ & $\begin{array}{l}43 a \\
42 a\end{array}$ & $\begin{array}{c}55 \mathrm{~b} \\
52 \mathrm{ab}\end{array}$ & $\begin{array}{c}49 \mathrm{ab} \\
56 \mathrm{~b}\end{array}$ & $\begin{array}{l}1,09 \mathrm{a} \\
1,08 \mathrm{a}\end{array}$ & $\begin{array}{l}1,10 \mathrm{a} \\
1,16 \mathrm{a}\end{array}$ & $\begin{array}{l}1,17 \mathrm{a} \\
1,15 \mathrm{a}\end{array}$ & $\begin{array}{l}0,34 \mathrm{a} \\
0,25 \mathrm{a}\end{array}$ & $\begin{array}{c}0,32 \mathrm{a} \\
0,38 \mathrm{ab}\end{array}$ & $\begin{array}{l}0,36 \mathrm{a} \\
0,39 \mathrm{~b}\end{array}$ \\
\hline VS2 & $\begin{array}{l}\mathrm{F} \\
\mathrm{M}\end{array}$ & $\begin{array}{l}40 a \\
47 a\end{array}$ & $\begin{array}{l}46 a \\
45 a\end{array}$ & $\begin{array}{l}40 a \\
40 a\end{array}$ & $\begin{array}{l}1,12 \mathrm{a} \\
1,15 \mathrm{a}\end{array}$ & $\begin{array}{l}1,11 \mathrm{a} \\
1,14 \mathrm{a}\end{array}$ & $\begin{array}{l}1,07 \mathrm{a} \\
1,10 \mathrm{a}\end{array}$ & $\begin{array}{l}0,34 \mathrm{a} \\
0,30 \mathrm{a}\end{array}$ & $\begin{array}{l}0,26 \mathrm{a} \\
0,33 \mathrm{a}\end{array}$ & $\begin{array}{l}0,27 \mathrm{a} \\
0,27 \mathrm{a}\end{array}$ \\
\hline $\mathrm{CF}$ & $\begin{array}{l}\mathrm{F} \\
\mathrm{M}\end{array}$ & $\begin{array}{l}286 a \\
280 a\end{array}$ & $\begin{array}{l}289 \mathrm{ab} \\
288 \mathrm{ab}\end{array}$ & $\begin{array}{l}310 \mathrm{~b} \\
305 \mathrm{~b}\end{array}$ & $\begin{array}{l}0,66 \mathrm{a} \\
0,90 \mathrm{~b}\end{array}$ & $\begin{array}{c}0,92 \mathrm{~b} \\
0,83 \mathrm{ab}\end{array}$ & $\begin{array}{c}0,88 \mathrm{ab} \\
0,70 \mathrm{a}\end{array}$ & $\begin{array}{c}2,09 \mathrm{ab} \\
2,50 \mathrm{~b}\end{array}$ & $\begin{array}{l}1,76 \mathrm{a} \\
1,84 \mathrm{a}\end{array}$ & $\begin{array}{l}2,25 b \\
1,67 a\end{array}$ \\
\hline \multicolumn{11}{|c|}{ Wistar Rats } \\
\hline $\mathrm{NC}$ & $\begin{array}{l}\mathrm{F} \\
\mathrm{M}\end{array}$ & $\begin{array}{c}42 \mathrm{ab} \\
42 \mathrm{a}\end{array}$ & $\begin{array}{l}50 \mathrm{~b} \\
49 a\end{array}$ & $\begin{array}{l}37 \mathrm{a} \\
50 \mathrm{a}\end{array}$ & $\begin{array}{l}1,10 \mathrm{a} \\
1,07 \mathrm{a}\end{array}$ & $\begin{array}{l}1,13 \mathrm{a} \\
1,14 \mathrm{a}\end{array}$ & $\begin{array}{l}1,10 \mathrm{a} \\
1,09 \mathrm{a}\end{array}$ & $\begin{array}{l}0,35 a \\
0,29 a\end{array}$ & $\begin{array}{l}0,35 a \\
0,37 a\end{array}$ & $\begin{array}{l}0,39 a \\
0,33 a\end{array}$ \\
\hline $\mathrm{TC}$ & $\begin{array}{l}\mathrm{F} \\
\mathrm{M}\end{array}$ & $\begin{array}{l}55 a \\
46 a\end{array}$ & $\begin{array}{l}48 a \\
49 a\end{array}$ & $\begin{array}{l}59 a \\
52 a\end{array}$ & $\begin{array}{l}1,13 \mathrm{a} \\
1,14 \mathrm{a}\end{array}$ & $\begin{array}{l}1,09 \mathrm{a} \\
1,17 \mathrm{a}\end{array}$ & $\begin{array}{l}1,14 \mathrm{a} \\
1,11 \mathrm{a}\end{array}$ & $\begin{array}{l}0,39 a \\
0,34 a\end{array}$ & $\begin{array}{l}0,37 \mathrm{a} \\
0,37 \mathrm{a}\end{array}$ & $\begin{array}{l}0,35 \mathrm{a} \\
0,32 \mathrm{a}\end{array}$ \\
\hline VS1 & $\begin{array}{l}\mathrm{F} \\
\mathrm{M}\end{array}$ & $\begin{array}{l}44 a \\
51 a\end{array}$ & $\begin{array}{l}56 \mathrm{~b} \\
58 \mathrm{a}\end{array}$ & $\begin{array}{c}50 \mathrm{ab} \\
56 \mathrm{a}\end{array}$ & $\begin{array}{l}1,11 \mathrm{a} \\
1,10 \mathrm{a}\end{array}$ & $\begin{array}{l}1,12 \mathrm{a} \\
1,15 \mathrm{a}\end{array}$ & $\begin{array}{l}1,07 \mathrm{a} \\
1,08 \mathrm{a}\end{array}$ & $\begin{array}{l}0,33 \mathrm{a} \\
0,37 \mathrm{a}\end{array}$ & $\begin{array}{l}0,35 a \\
0,36 a\end{array}$ & $\begin{array}{l}0,34 \mathrm{a} \\
0,42 \mathrm{a}\end{array}$ \\
\hline VS2 & $\begin{array}{l}\mathrm{F} \\
\mathrm{M}\end{array}$ & $\begin{array}{l}49 a \\
44 a\end{array}$ & $\begin{array}{l}51 \mathrm{a} \\
45 \mathrm{a}\end{array}$ & $\begin{array}{l}53 a \\
49 a\end{array}$ & $\begin{array}{l}1,07 \mathrm{a} \\
1,13 \mathrm{a}\end{array}$ & $\begin{array}{l}1,12 \mathrm{a} \\
1,16 \mathrm{a}\end{array}$ & $\begin{array}{l}1,11 \mathrm{a} \\
1,07 \mathrm{a}\end{array}$ & $\begin{array}{l}0,32 \mathrm{a} \\
0,33 \mathrm{a}\end{array}$ & $\begin{array}{l}0,36 \mathrm{a} \\
0,32 \mathrm{a}\end{array}$ & $\begin{array}{l}0,37 \mathrm{a} \\
0,31 \mathrm{a}\end{array}$ \\
\hline $\mathrm{CF}$ & $\begin{array}{l}\mathrm{F} \\
\mathrm{M}\end{array}$ & $\begin{array}{l}285 a \\
299 a\end{array}$ & $\begin{array}{l}296 a \\
300 a\end{array}$ & $\begin{array}{l}295 a \\
295 a\end{array}$ & $\begin{array}{l}0,87 \mathrm{~b} \\
0,83 \mathrm{a}\end{array}$ & $\begin{array}{l}0,90 \mathrm{~b} \\
0,78 \mathrm{a}\end{array}$ & $\begin{array}{r}0,60 \mathrm{a} \\
0,80 \mathrm{a}\end{array}$ & $\begin{array}{l}1,94 \mathrm{a} \\
2,09 \mathrm{a}\end{array}$ & $\begin{array}{l}2,04 \mathrm{a} \\
2,00 \mathrm{a}\end{array}$ & $\begin{array}{l}2,05 \mathrm{a} \\
2,06 \mathrm{a}\end{array}$ \\
\hline
\end{tabular}

Inducation. NC - Negative Control. TC - Technique Control. VS1 - Vehicle Substance 1. VS2 - Vehicle Substance 2. CF - Cyclophosphamide. O - number of observers. PE - Polychromatic Erythrocytes. NE Normochromatic Erythrocytes. MN - Micronucleus. Count in 2000 cells/animal of 10 animal/group/sex/line. ${ }^{\mathrm{T}}$ Analyzed variable using the ANOVA test (X: average; SD: Standard Deviation, $\mathrm{p}<0,05$ ). RAnalyzed variable using the $\chi^{2}$ non parametric test $(p<0,01)$. a, b - Different letters it differs when comparing the results of each observer in the same variable, rat line, sex and experimental group. 
$0.15 \pm 0.06 \%$ of polychromatic erythrocytes with micronucleus. The response of Sprague Dawley line to the cyclophosphamide was high, but smaller than the clastogenicity results obtained in both sexes of Lewis and Wistar lines. The total MN level in Sprague Dawley rats was lower than in animals of other lines. On the other hand, the cyclophosphamide revealed its biggest clastogenic effect in the males of Wistar rats [11, 16]. Significant differences between sexes were not observed. The different response of rat lines on cyclophosphamide is the result of their differences in drug metabolism [7, 17, 18]. Hepatic enzymes are fundamental in the first phase of xenobiotic metabolism that participates in the cyclophosphamide metabolism in the liver [7, 11, 17-21]. The differences in expressions of enzymes lead to differences in their metabolisms $[20,21]$; different concentrations of the metabolites, active mutagene levels and so on.

The comparison of data of three independent observers is shown in the Table 2. In general the results demonstrated little variation of the variables and studied experimental groups. These data are in good correspondence with results of other investigators [23, 24] where high repeatability and consistency in their micronucleus assay in bone marrow cells were demostrated. Thus, it may be recommended to explore genotoxic effects of drugs at the chromosome level [9].

Being based on the data obtained, we suppose that the Sprague Dawley rat line is more genetically stable than the other lines. This line revealed the lower spontaneous indexes and acceptable cyclophosphamide-induced indexes [25, 26]. These results are important also due to heterogeneity of response of the Sprague Dawley rats, similar to those in human populations [25, 26]. Futher, we suggest to use Wistar rats in experiments with cyclophosphamide intraperitoneal treatment as positive control. In our experiments Wistar line was the most sensitive to the mutagenic action of cyclophosphamide and this line may be good biomodel to detection of mutagenic effects of similar substances.

Conclusions. The Sprague Dawley rats of both sexes differ significantly from the other lines in lower spontaneous indexes and acceptable induced with cyclophosphamide indexes in the bone marrow cells micronucleus assay.
The authors thank to the technical personnel of the animal facilities installation from Research and Development Vicepresidency of the Finlay Institute, Cuba.

\section{D.F. Arencibia-Arrebola, L.A. Rosario-Fernandez, Y.E. Suarez-Fernandez, A. Vidal-Novoa \\ СРАВНЕНИЕ ЧАСТОТЫ МИКРОЯДЕР В КЛЕТКАХ КОСТНОГО МОЗГА ТРЕХ ЛИНИЙ КРЫС}

Сравнивали спонтанные и индуцированные циклофосфамидом микроядерные индексы в клетках костного мозга трех линий крыс - Sprague Dawley, Льюис и Вистар. Сформировали пять экспериментальных групп (по 10 животных каждого пола и линии в каждой группе). Первую группу использовали в качестве негативного контроля (интактные животные), вторая служила контролем влияния перорального приема препаратов. Все условия содержания были одинаковыми для всех групп животных. Крыс третьей группы обрабатывали $2 \%$ Tween 65, а четвертой группы $0,9 \% \mathrm{NaCl}$. Оба вещества вводили перорально в дозе 2 мл/кг в течение 14 дней. Животных пятой группы обрабатывали внутрибрюшинно циклофосфамидом в дозе 50 мг/кг (10 мл/кг раствора), за 48 и 24 ч до эвтаназии. Линия Sprague Dawley (оба пола) существенно отличается от других. Крысы этой линии имели низкие показатели спонтанного образования микроядер и высокий индекс при индукции циклофосфамидом, а также процент эритроцитов с микроядрами в костном мозге и индекс цитотоксичности. Полученные результаты позволяют определить наиболее подходящие линии крыс в качестве модельных животных для изучения генотоксичности. Это также позволит получать более точные оценки генотоксичности различных веществ.

\section{D.F. Arencibia-Arrebola, L.A. Rosario-Fernandez, Y.E. Suarez-Fernandez, A. Vidal-Novoa \\ ПОРІВНЯННЯ ЧАСТОТИ МІКРОЯДЕР В КЛІТИНАХ КІСТКОВОГО МОЗКУ ТРЬОХ ЛІНІЙ ЩУРІВ}

Порівнювали спонтанні та індуковані циклофосфамідом мікроядерні індекси в клітинах кісткового мозку трьох ліній щурів - Sprague Dawley, Люіс і Вистар. Сформували п'ять експериментальних груп (по 10 тварин кожної статі i лінії в кожній групі). Першу групу використали як негативний контроль (інтактні тварини), друга слугувала контролем впливу перорального прийому препаратів. Всі умови утримання були однаковими для всіх груп тварин. Щурів третьої групи

ISSN 0564-3783. Цитология и генетика. 2013. № 2 
обробляли $2 \%$ Tween 65, а четвертої групи $0,9 \% \mathrm{NaCl}$. Обидві речовини вводили перорально в дозі 2 мл/кг впродовж 14 днів. Тварин п'ятої групи обробляли внутрішньочеревно циклофосфамідом в дозі 50 мг/кг (10 мл/кг розчину), за 48 і 24 год до евтаназії. Лінія Sprague Dawley (обидві статі) істотно відрізнялась від інших. Щури цієї лінії мали низькі показники спонтанного утворення мікроядер і високий індекс при індукції циклофосфамідом, а також відсоток еритроцитів 3 мікроядрами в кістковому мозку та індекс цитотоксичності. Отримані результати дозволяють визначити найбільш відповідні лінії щурів як модельних тварин для вивчення генотоксичності. Це також дозволить отримувати точніші оцінки генотоксичності різних речовин.

\section{REFERENCES}

1. Arencibia D.F., Rosario L.A., Suárez Y.E., Vidal A. Letter to the Editor: Assessment and comparison between three lines of rats in four genotoxicity assay. Retel 2011; 35(2):16-24.

2. OECD. Genetic Toxicology: In vivo Mammalian Erythrocyte Micronucleus Test, in bone marrow cells TG 474 (Annual Report 2009). Public Affairs and Communications Directorate Editions. Paris. France: OECD online Bookshop Editions. 2009. p. 7-123. Available in: http://www.oecd.org/ bookshop

3. Yiqiang L., Mengmeng Q., Liwei S., Yulin W., Yuangao C.H. Genotoxicity study of phenol and cresol using the micronucleus test and the comet assay. Toxicology \& Environmental Chemical 2005; 87(3):365-372.

4. Matuo R., Oliveira R.J., Silva A.F., Mantovani M.S. Ribeiro L.R. Anticlastogenic Activity of Aqueous Extract of Agaricus blazei in Drug-Metabolizing Cells (HTCs) During Cell Cycle. Toxicology Mechanisms Method 2007; 17(3):147-152.

5. CCAC. Canadian Council on Animal Care. Guidelines for the use of animals in Psychology. In: Olfert E.D. Cross B.M. McWilliam D.V.M. McWilliam A.A. (Eds.) Ottawa : Bradda Printing Services Inc. 1997.p. 155-162.

6. Arruzazabala M.L., Mas R., Molina V. Effect of D-004, a lipid extracts from the Cuban royal palm fruit on atypical prostate hyperplasia induced by phenylephrine in rats. Drugs in R\&D 2006; 7:233-241.

7. Shayne C.G. Animal Models in toxicology. In: Published by Shayne C. Gad and Taylor \& Francis Group. Toxicology: Chapter 2 and 3. The Mouse and Rats. 2nd edition. New York, 2007. p. 24162.

8. Arencibia D.F., Rosario L.A. Evaluation of Sprague
Dawley rats as experimental animal models in the micronuclei and chromosomal aberration assays in bone marrow cells. Veterinary Argentinean Journal 2010; 27(264):1-13.

9. Hayashi M., Tice R., Macgregor J.T. In vivo rodent erythrocyte micronucleus assay. Mutat. Res. 1993; 5:120-134.

10. Arencibia D.F., Rosario L.A., Morffi J., Curveco D. Development and standardization of the three genotoxicity assays technique. Retel 2009; 25(3): 22-38.

11. Gocke E., Bürgin H., Müller L. Literature review on the genotoxicity, reproductive toxicity, and carcinogenicity of ethyl methanesulfonate. Toxicology Letters 2009; 190:254-265.

12. Arencibia D.F., Rosario L.A., Suárez Y.E., Vidal A. Comparison between two murine biomodels in the micronuclei and chromosomal aberration assay in bone marrow cell. Quimica Viva 2011; 10(2):106117.

13. Arencibia D.F., Rosario L.A., Suárez Y.E., Delgado L. Spontaneous and induced frequency of transplacental micronucleus in Sprague Dawley rats. Invet $2011 ; 13(1): 6-15$.

14. Arencibia D.F., Rosario L.A., Suárez Y.E., Vidal A. Assessment of Sprague Dawley rats as biomodel in two antigenotoxicity assays. Veterinary World 2011; 4(10):450-460.

15. Gymez B.C., Zuciga G., Zamora A., Ramos M.L., Batista C.M., Torres B.M. Folate supplementation of cyclophosphamide-treated mothers diminishes micronucleated erythrocytes of peripheral blood of newborn rat. Environmental Molecular Mutagenesis 2004; 4:174-178.

16. Zuciga G., Torres O., Zamora A., Gymez B.C., Ramos M.L., Martinez S., González A., Lunas J., Ramos A., Ontiveros D., Gallegos M.P. Differences in number of micronucleated erythrocytes among young and adult animals including humans. Spontaneous micronuclei in 43 species. Mutation Research 2001; 494:161-167.

17. Amri H., Batt A., Siest G. Comparison of cytochrome $\mathrm{P}-450$ content and activities in liver microsomes of seven species including man. Xenobiotica 1986; 16: 351-358.

18. Jana N.R., Sarkar S., Yonemoto J. Strain differences in cytochrome P451A1 gene expression caused by 2,3,7,8-tetrachlorordibenzo-p-dioxin in the rat liver: Role of the aryl hydrocarbon receptor and its nuclear translocator. Biochem. Biophys. Res. Commun 1998; 248:554-558.

19. Santiago C., Bandrŭs F., Gymez F. Cytochrome P-450 polymorphisms : Their role as biological Markers. Works Medicine 2002; 2:130-140.

20. Quicones L., Rosero M., Roco A., Moreno I., Sasso 
J., Varela $N$. Role of cytochrome P450 enzymes in the metabolism of antineoplastic drugs. Rev Med Chile 2008; 136:1327-1335.

21. Solus J.F., Arietta B.J., Harris J.R., Sexton D.P., Steward J.Q., Mcmunn C. Genetic variation in eleven phase I drug metabolism genes in an ethnically diverse population. Pharmacogenomics 2004; 5:895-931.

22. Greggi L., Araújo M.C., D’Arc C.J., Bianchi M.L. Effects of the antioxidants curcumin and vitamin $\mathrm{C}$ on cisplatin-induced clastogenesis in Wistar rat bone marrow cells. Mutation Research 2000; 465(1-2):131-137.

23. Zalacain M., Sierrasesúmaga L., Patiño A. The cytogenetic assay as a measure of genetic instability induced by genotoxic agents. An Sist Sanit Navar 2005; 28(2):227-236.

24. Bowen D., Whitwell J., Lillford L., Henderson D., Kidd D., Garry S., Pearce G., Beevers C., Kirkland D.
Evaluation of a multi-endpoint assay in rats, combining the bone-marrow micronucleus test, the Comet assay and the flow-cytometric peripheral blood micronucleus test. Mutation Research; 2011; 722(1):7-19.

25. Torous D., Dertinger S., Hall N., Tometsko C. Enumeration of micronucleated reticulocytes in rat peripheral blood: a flow cytometric study. Mutation Research 2000; 465:91-99.

26. MacGregor J.T., Bishop M.E., McNamee J.P., Hayashi M., Asano N., Wakata A., Nakajima M., Saito J., Aidoo A., Moore M.M., Dertinger S.D. Flow cytometric analysis of micronuclei in peripheral blood reticulocytes : 2 . An efficient method of monitoring chromosomal damage in the rat. Toxicological Sciences 2006; 94(1):92-107.

Received 06.07.11 